\title{
Atividade física e desempenho em tarefas de funções executivas em idosos
} saudáveis: dados preliminares

\author{
Physical activity and performance in executive function tasks in healthy older adults: preliminary data
}

\section{Andreza Giuliane Guimarães Moreira¹, Leandro Fernandes Malloy-Diniz², Daniel Fuentes³, Humberto Correa ${ }^{4,5}$, Guilherme Menezes Lage6}

1 Terapeuta ocupacional, especialista em orientação e mobilidade do Centro Municipal de Atenção Domiciliar em Saúde (Cemads), Nova Lima, MG.

2 Professor doutor do Departamento de Psicologia da Faculdade de Filosofia e Ciências Humanas da Universidade Federal de Minas Gerais (Fafich/UFMG).

${ }_{3}^{3}$ Professor doutor da Unidade de Psicologia e Neuropsicologia do Instituto de Psiquiatria do Hospital das Clínicas da Faculdade de Medicina da Universidade de São Paulo (IPq-HCFMUSP.

${ }_{4}^{4}$ Professor doutor do Serviço de Psiquiatria do Hospital das Clínicas da UFMG.

5 Professor doutor do Departamento de Farmacologia do Instituto de Ciências Biológicas da UFMG.

${ }_{6}^{6}$ Professor mestre da Faculdade de Ciências da Saúde da Universidade FUMEC. Doutorando em Neurociências pela UFMG.

\section{Resumo}

Contexto: A atividade física parece exercer um efeito positivo sobre vários processos cognitivos em idosos. Entretanto, pouco se sabe sobre o impacto da atividade física sequencial, como a dança, sobre as funções executivas dos idosos. Objetivo: Avaliar a relação entre o tipo de atividade física praticada e o desempenho em tarefas de funções executivas em idosos. Métodos: Participaram do estudo 35 idosos entre 60 e 69 anos de idade. O grupo controle (GC) foi composto por idosos sedentários; o grupo ativos (GA) foi composto por idosos praticantes de atividade física; o grupo dança (GD) foi composto por idosos praticantes de atividade física idêntica ao GA acrescido da prática de dança sênior. Após uma triagem inicial, todos os participantes foram submetidos a testes de funções executivas. Resultados: Comparado aos demais grupos, o GD apresentou melhor desempenho em termos de (1) planejamento, medida essa observada no teste da Torre de Londres e (2) controle inibitório, por meio do teste de Stroop. Conclusões: Os resultados preliminares encontrados sugerem uma especificidade da atividade física sobre o desempenho de determinadas funções executivas. A perspectiva é de que com uma maior amostra esses achados sejam mantidos.

Moreira AGG, et al. / Rev Psiq Clín. 2010;37(3):109-12

Palavras-chave: Cognição, atividade física, dança, saúde, envelhecimento.

\begin{abstract}
Background: Physical activity seems to produce positive effects on many cognitive processes in elderly people. Nevertheless, little is known about the impact of sequential physical activity, such as dancing, on the executive functions in older adults. Objective: To evaluate, in elderly people, the relation between the type of physical practice and the performance in executive functions. Methods: Thirty five older adults, age 60-69, participated in this study. The control group (CD) was composed by sedentary participants; the physical activity group (PAG) was composed by practitioners of physical activity; the dance group (DG) was composed by practitioner of identical physical activity to the PAG plus practice of senior dance. After an initial triage, executive function tests were administered for all participants. Results: Compared to the other groups, the DG presented better performance in the measures of planning (Tower of London) and inhibitory control (Stroop test). Discussion: The preliminary findings suggest a specificity of physical activity on the performance of some executive functions. Our perspective is to find the same results with a larger sample.
\end{abstract}

Moreira AGG, et al. / Rev Psiq Clín. 2010;37(3):109-12

Keywords: Cognition, physical education and training, dancing, health, aging.

\section{Introdução}

Os impactos positivos da prática regular de exercícios físicos para a saúde são evidenciados na literatura ${ }^{1,2}$. Somados aos inúmeros benefícios relacionados à saúde em geral, a atividade física parece exercer um efeito positivo sobre vários processos cognitivos em idosos ${ }^{3}$. A maior parte dos estudos que aborda esse tema tem focado no potencial angiogênico de atividades cíclicas aeróbias como o andar e o correr. Enquanto alguns resultados de estudos mostram uma relação positiva entre atividade física aeróbia e cognição $\mathrm{O}^{4,5}$, outros achados sugerem que a capacidade aeróbia não é um bom mediador da relação atividade física e cognição em idosos ${ }^{6,7}$. Fatores de influência na inconsistência desses achados podem estar relacionados ao tipo de atividade física analisada, assim como às medidas utilizadas para definição da intensidade de exercício ${ }^{8}$.

Além do foco sobre a capacidade aeróbia, é possível direcionar os esforços de pesquisa para outras variáveis psicológicas e fisiológicas de influência nessa relação ${ }^{7}$. Atividades como o caminhar e o correr com o objetivo de melhoria cardiorrespiratória requerem menor participação de funções executivas quando comparadas às atividades sequenciais que exigem planejamento, tomada de decisão e monitoramento detalhado das ações, funções essas observadas nos esportes $\operatorname{coletivos}^{9} \mathrm{e}$ na dança ${ }^{10}$. Nesse sentido, a atividade física que tenha como característica a estimulação cognitiva pode exercer um efeito positivo sobre as funções executivas, que correspondem a um conjunto de habilidades que, de forma integrada, permitem ao indivíduo dirigir comportamentos a metas, avaliar eficiência e a adequação desses comportamentos, abandonar estratégias ineficazes em prol de outras mais eficientes e, desse modo, resolver problemas imediatos, de médio e de longo prazo ${ }^{11}$. Pouco tem sido discutido em relação a essa hipótese de que a natureza da atividade física apresente um papel de maior influência no desempenho cognitivo do que a estimulação cardiorrespiratória, uma das exceções encontradas na literatura é o estudo de Hogan ${ }^{12}$.

Hogan ${ }^{12}$ propõe que atividades físicas que apresentem em sua natureza uma maior demanda cognitiva, tais como aquelas que 
exigem memorização de sequências de execução de padrões de movimentos e atenção sustentada para alterações intencionais dos movimentos, apresentem benefícios não somente aos sistemas musculoesquelético e cardiovascular, mas também ao sistema cognitivo por meio do engajamento em processos de controle executivo. A ativação da área pré-frontal medial do córtex em associação com o estriado ventral está mais relacionada à execução de tarefas realizadas em sequências fixas e repetitivas como em tarefas cíclicas, guiadas internamente (por exemplo, caminhada). Por outro lado, tarefas guiadas externamente (por exemplo, controle motor dependente de um ritmo musical e das ações de um parceiro) parecem estar associadas com a ativação da região pré-frontal anterolateral e do estriado dorsolateral, áreas mais envolvidas em funções de planejamento e gerenciamento ${ }^{13}$. Essas especializações podem refletir diferenciações funcionais que repercutem reciprocamente no controle motor e nos processos executivos.

Pouco se sabe sobre o impacto da atividade física sequencial, como a dança, sobre a cognição dos idosos. Mais especificamente para os idosos, a dança sênior é uma atividade física que requer planejamento, tomada de decisão e monitoramento, tarefas estas relacionadas às funções executivas. Trata-se de um conjunto sistematizado de coreografias baseado em danças folclóricas de diferentes culturas, especialmente adaptadas às possibilidades e às necessidades da pessoa idosa ${ }^{14}$. Em uma tentativa de avaliar a relação entre o tipo de atividade física prescrita e o desempenho em tarefas de funções executivas, comparamos os dados preliminares sobre o desempenho cognitivo de idosos que participam de diferentes programas de atividade física.

\section{Materiais e métodos}

\section{Participantes}

Foi conduzido um estudo transversal no qual participaram 35 idosos entre 60 e 69 anos de idade $(\bar{x}=64,4 \pm 3,3)$ que vivem de forma autônoma (escolaridade média de 7,4 $\pm 4,6$ anos). Trata-se de uma amostra não probabilística e intencional na qual os participantes compuseram três grupos. O grupo controle $(\mathrm{GC})(\mathrm{n}=11)$ foi composto por idosos sedentários, sendo dez mulheres e um homem. O grupo ativos (GA) $(\mathrm{n}=12)$ foi composto por onze mulheres e um homem, sendo todos idosos praticantes de atividade física (caminhada, alongamento e exercícios calistênicos). O grupo dança (GD) $(n=12)$ foi composto por onze mulheres e um homem, sendo todos idosos praticantes de atividade física idêntica ao GA acrescido da prática de dança sênior. $\mathrm{O}$ estudo foi aprovado por um comitê de ética em pesquisa local e todos participaram com consentimento livre e esclarecido.

Os idosos do GA e do GD participavam de dois grupos de terceira idade de Nova Lima, uma cidade da região metropolitana de Belo Horizonte. Apesar de não haver controle da intensidade da caminhada em ambos os grupos, a frequência semanal e a duração de cada sessão eram idênticas entre os grupos, duas vezes por semana durante 45 minutos. Os exercícios calistênicos eram realizados por ambos os grupos duas vezes por semana por meio de movimentos que exigiam contração isométrica e isotônica concêntrica. Em média a duração desses exercícios era de 15 minutos realizados antes ou depois da atividade aeróbia (caminhada). A exceção no que tange às atividades praticadas é que os participantes do GD praticavam uma sessão semanal extra de 50 minutos de dança sênior oferecida por uma terapeuta ocupacional. A atividade de dança sênior vinha sendo oferecida há seis meses para os participantes.

Nenhum dos participantes informou fazer uso de medicamentos que interferissem no funcionamento cognitivo e motor ou apresentar doenças neurológicas, psiquiátricas ou musculoesqueléticas que interferissem no desempenho dos testes.

\section{Procedimentos}

Os sujeitos foram avaliados em duas etapas. Inicialmente foi realizada triagem de aspectos globais da cognição e do humor dos sujeitos dos três grupos. Tal procedimento teve como objetivo verificar a homogeneidade de tais variáveis entre os grupos. Em seguida, os sujeitos foram submetidos à avaliação específica das funções executivas. Os testes foram aplicados em uma sala para que os participantes sofressem o mínimo impacto de interferências externas.

Na triagem dos sintomas depressivos e declínio cognitivo patológico, todos os participantes foram submetidos ao Consortium to Establish a Registry for Alzheimer's Disease (CERAD) e ao Geriatric Depression Scale (GDS). Nenhum dos idosos apresentou escore indicativo de declínio cognitivo ou depressão.

Após a triagem inicial, todos os sujeitos foram submetidos aos seguintes testes de funções executivas ${ }^{15}$ : Fluência Verbal Fonológica (FAS), Fluência Verbal Semântica (categoria animais), Stroop Color Word Test (SCWT), Fluência de Desenhos e teste da Torre de Londres.

No teste de FAS é pedido ao sujeito que fale a maior quantidade de palavras iniciadas com as letras F, A e S durante o período de 1 minuto (para cada uma das letras). É computada a quantidade total de palavras somando-se todas as letras (exceto erros e repetições). O teste de fluência verbal semântica é semelhante ao FAS. No entanto, nesse teste, os sujeitos devem falar a maior quantidade de palavras pertencentes à categoria animais durante um minuto. Novamente é computada a quantidade total de palavras proferidas com exceção das repetições.

O SCWT é composto por três etapas. Na primeira, é apresentado ao sujeito um cartão contendo colunas de retângulos coloridos (amarelo, vermelho, verde ou azul), os quais devem ser nomeados o mais rápido possível pelo probando. Na segunda parte, é apresentado ao sujeito um novo cartão contendo palavras aleatórias coloridas com as cores supracitadas. Novamente, o sujeito deve dizer o nome das cores o mais rápido que conseguir. Na última etapa, é apresentado ao sujeito um novo cartão com nomes de cores, cada qual colorido com uma cor incongruente (por exemplo, palavra verde colorida de vermelho). Assim como nas etapas anteriores, o sujeito deve dizer qual a cor com a qual a palavra foi impressa, inibindo a tendência de ler o nome da cor. Nas três etapas, são mensurados a quantidade de erros de nomeação de cores e o tempo total de nomeação de todos os elementos do cartão.

No teste de Fluência de Desenhos, os sujeitos recebem uma folha contendo 40 retângulos idênticos, cada um contendo 5 pontos. Cabe ao sujeito fazer desenhos diferentes unindo os pontos dentro de cada retângulo. São medidas no teste a quantidade de desenhos inéditos, a quantidade de repetições de desenhos (erros perseverativos) e a quantidade de desenhos errados.

No teste da Torre de Londres, é usada uma base de madeira com três hastes de tamanhos diferentes em que estão três bolas de cores diferentes (vermelho, verde e azul). Na versão original desenvolvida por Shallice ${ }^{16}$ e adaptada posteriormente por Kricorian et al. ${ }^{17}$ são apresentados ao sujeito 12 problemas em ordem crescente de dificuldade, um de cada vez, que devem ser resolvidos movimentando as bolas nas hastes. Os problemas são apresentados por intermédio de cartões nos quais estão diferentes configurações envolvendo a disposição das esferas nas hastes. O sujeito deve realizar a tarefa com a menor quantidade possível de movimentos que conseguir. Cada problema apresenta uma quantidade mínima de movimentos que pode variar de 2 a 5 movimentos. Para cada tentativa, o sujeito tem três chances de resolver com a quantidade mínima de movimentos. Se o probando resolver da primeira vez com a quantidade mínima de movimentos, ganha três pontos. Na segunda tentativa, ganha dois pontos e na terceira, um ponto. Caso não consiga resolver em nenhuma tentativa, não ganha os pontos do item. A pontuação máxima obtida no teste é de 36 pontos.

\section{Análise dos dados}

O teste de Kolmogorov-Smirnov foi aplicado para verificar a normalidade dos dados. Para os dados com distribuição normal, foi utilizada a ANOVA one-way e para análise post hoc, o teste de Tukey ( $\mathrm{p}<$ 0,05 ), assim como o teste $t$ de Student para análise entre duas médias. 
Para as demais medidas, foi utilizado o teste de Kruskal-Wallis e para análises post hoc, o teste U de Mann-Whitney com ajuste de $p$ pelo método de Bonferroni $(\mathrm{p}<0,01)$.

\section{Resultados}

As análises das medidas demográficas mostraram que, para a média de escolaridade dos grupos, (1) controle $=6,82$ anos, (2) ativos $=7,92$ anos e (3) dança $=7,50$ anos, não houve diferença significativa $[\mathrm{H}(2$, $35)=0,57, p=0,74]$. Em relação às médias de idade dos grupos, (1) sedentário $=64,09$ anos, $(2)$ ativo $=64,08$ anos e (3) dança $=64,92$ anos, também não houve diferença significativa $[\mathrm{F}(2,32)=0,23, \mathrm{p}=$ $0,79]$. Para a média de tempo de prática entre os grupos ativo $(3,43$ anos) e dança (3,02 anos), o teste $t$ de Student não indicou diferença significativa entre grupos $[t(\mathrm{DF}=22)=0,75, \mathrm{p}=0,46]$.

Nas análises comparativas do desempenho cognitivo entre grupos, foi encontrado um melhor desempenho do GD comparado aos demais nas medidas de número de erros na parte III do teste de Stroop $[\mathrm{H}(2,35)=9,24 ; \mathrm{p}=0,009]$ e escore total no teste de Torre de Londres $[F(2,32)=5,74, p=0,007]$. Para as outras medidas as diferenças não foram significativas (Tabela 1 ).

Tabela 1. Escores dos grupos em cada uma das medidas neuropsicológicas e valor de significância encontrada

\begin{tabular}{l|c|c|c|c}
\hline \multirow{2}{*}{ Medidas } & \multicolumn{4}{c}{ Grupos } \\
\cline { 2 - 5 } \multicolumn{1}{c}{ GC } & GA & GD & \multicolumn{1}{c}{$p$ level } \\
\hline $\begin{array}{l}\text { Fluência verbal fonológica (total de } \\
\text { palavras) }\end{array}$ & 29,81 & 31,33 & 37,25 & 0,32 \\
\hline $\begin{array}{l}\text { Fluência verbal semântica (total de } \\
\text { palavras) }\end{array}$ & 13,36 & 15,16 & 15,41 & 0,48 \\
\hline $\begin{array}{l}\text { Fluência de desenhos (repetições) })^{n p} \\
\text { Fluência de desenhos (erros)np }\end{array}$ & 1,55 & 3,18 & 1,33 & 0,12 \\
\hline $\begin{array}{l}\text { Fluência de desenhos (total de desenhos } \\
\text { corretos) }\end{array}$ & 15,09 & 18,91 & 16,75 & 0,29 \\
\hline Stroop parte III (tempo em segundos) $n p$ & 38,18 & 40,5 & 37,83 & 0,91 \\
\hline Stroop parte III (erros)np & 3,36 & 3,66 & 0,16 & 0,009 \\
\hline Torre de Londresp & 30,36 & 30,5 & 33,75 & 0,007 \\
\hline
\end{tabular}

* $p$ = análise paramétrica; ${ }^{n p}=$ análise não paramétrica. GC: grupo controle; GA: grupo ativos; GD: grupo dança.

\section{Discussão}

Os resultados encontrados sugerem uma especificidade da atividade física sobre o desempenho de determinadas funções executivas. É possível que o nível de exigência perceptomotora da atividade praticada possa influenciar a cognição ${ }^{18}$. Nesse caso, uma atividade cíclica como o caminhar requer menor participação de funções executivas quando comparada às atividades motoras sequenciais guiadas externamente como a dança.

Comparado aos demais grupos, o GD apresentou melhor desempenho em termos de planejamento, medida essa observada no teste da Torre de Londres, e controle inibitório, pelo teste de Stroop. Essa especificidade na relação atividade física e cognição pode estar ligada à ativação de áreas cerebrais específicas. Processos frontais relacionados ao controle executivo e ao controle motor parecem seguir princípios organizacionais comuns ${ }^{13}$; principalmente em tarefas motoras guiadas externamente que são mediadas pela região pré-frontal anterolateral e pelo estriado dorsolateral, áreas mais envolvidas em funções de planejamento e gerenciamento ${ }^{13}$.

A atividade que diferenciou os grupos que participavam de programas de atividade física foi a dança, atividade essa que requer planejamento, tomada de decisão e monitoramento on-line das ações, principalmente na dança sênior onde o curso das ações motoras depende de coordenação inter-sujeitos ${ }^{14}$, o que demanda constantes ajustes para que as metas de espaço e tempo sejam alcançadas. Ao nosso conhecimento, somente um estudo investigou a relação entre a prática da dança e o desempenho em funções executivas ${ }^{19}$.
Ao contrário do presente estudo, Verghese ${ }^{19}$ não encontrou diferença no desempenho de funções executivas entre idosos que praticavam dança e idosos que não praticavam dança.

Algumas possíveis explicações podem ser apresentadas para essa discrepância entre os resultados encontrados no presente estudo e os resultados de Verghese ${ }^{19}$. No estudo de Verghese ${ }^{19}$, observa-se um baixo valor médio (e alta variabilidade) de quantidade de prática dos participantes, que foi de 4,3 \pm 3 dias por mês. Outro fator é que não houve um maior controle sobre o tipo de atividade praticada. Os participantes praticavam diferentes tipos de dança e sem orientação de um profissional, sendo que $30 \%$ deles não praticavam nenhum tipo de dança específica. É possível que alguns desses participantes não tenham obtido os benefícios da dança sobre o desempenho cognitivo em função do baixo volume e da frequência de prática e também da falta de regularidade de atividades que requeriam o mesmo tipo de exigência cognitiva. Um último aspecto a ser destacado é a não utilização de testes que medeiam planejamento e controle inibitório. Se a especificidade entre atividade física e funções executivas encontrada no presente estudo for um fenômeno robusto, é possível que as diferenças entre grupos de praticantes e não praticantes sejam encontradas em testes que exijam funções específicas. Uma comparação que fortalece essa hipótese é a de que no presente estudo e no de Verghese ${ }^{19}$ não foi encontrada diferença entre grupos nos testes de fluência verbal.

Partindo da hipótese de que os efeitos da atividade física aeróbica via angiogênese levam ao aumento da ativação de áreas pré-frontais e parietais que estão envolvidas no controle executivo ${ }^{4}$, poderia ser esperado um desempenho superior do grupo ativo em relação ao GC. Resultados de estudos ainda são controversos em relação aos benefícios da atividade física aeróbia sobre a cognição. Enquanto estudos observacionais tendem a demonstrar efeitos positivos dessa relação ${ }^{20,21}$, estudos de intervenção nem sempre encontram essa relação positiva ${ }^{22,23}$. Suporte para a hipótese de que ganhos na capacidade aeróbia não estão relacionados a ganhos em funções cognitivas foi encontrado tanto em um estudo de metarregressão ${ }^{7}$ quanto em um estudo de metanálise ${ }^{15}$. Como descrito por Etnier et al.7 " "nosso foco sobre a aptidão aeróbia pode ter sido equivocado" (p. 126). Os resultados encontrados nos permitem ao menos alertar os profissionais da saúde que prescrevem atividade física para grupos de idosos que somente a atividade física aeróbia de baixa intensidade e sem controle da magnitude do estímulo pode não surtir efeitos sobre algumas funções executivas em idosos.

É importante ressaltar as limitações do presente estudo por ser observacional de delineamento transverso, com uma pequena amostra e com controle somente das variáveis tempo de prática, duração da atividade e frequência semanal de atividades. Como o agrupamento dos indivíduos foi feito de modo intencional e não randomizado, não há como se afirmar que o efeito da dança sobre as funções executivas tenha sido controlado. Comparado aos participantes dos grupos sedentário e ativos, é possível que os idosos do GD já apresentassem maior nível de desempenho em funções executivas antes mesmo do início da prática da atividade física. Sugerem-se estudos longitudinais e análise da capacidade aeróbica dos participantes. Futuras investigações poderiam comparar, por intermédio do método experimental, grupos de idosos que praticam atividades físicas que requeiram altos níveis de controle executivo e que sejam guiadas externamente, como os esportes coletivos e diferentes modalidades de dança, com atividades cíclicas guiadas internamente, como a corrida e a natação.

\section{Referências}

1. Assunção SS, Cordás TA, Araújo LA. Atividade física e transtornos alimentares. Rev Psiq Clín. 2002;29(1):4-13.

2. Teixeira PC, Costa RF, Matsudo SMM, Cordás TA. A prática de exercícios físicos em pacientes com transtornos alimentares. Rev Psiq Clín. 2009;34(4):145-52.

3. Hillman CH, Motl RW, Pontifex MB, Posthuma D, Stubbe JH, Boomsma DI, et al. Physical activity and cognitive function in a cross-section of younger and older community-dwelling individuals. Health Psych. 2006;25(6):678-87. 
4. Kramer AF, Sowon H, Cohen NJ, Banich MT, McAuley E, Harrison JC, et al. Ageing, fitness and neurocognitive function. Nature. 1999;400:418-19.

5. Themanson JR, Hillman CH, Curtin JJ. Age and physical activity influences on action monitoring during task switching. Neurob Aging. 2006;27:1335-45.

6. Bunce D, Murden F. Age, aerobic fitness, executive function and episodic memory. Eur J Cog Psych. 2006;18(2):221-33.

7. Etnier JL, Nowell PM, Landers DM, Sibley BA. A meta-regression to examine the relationship between aerobic fitness and cognitive performance. Brain Res Rev. 2006;52:119-30.

8. Newson RS, Kemps EB. The influence of physical and cognitive activities on simple and complex cognitive tasks in older adults. Exp Aging Res. 2006;32(3):341-62.

9. Williams AM, Ericsson KA. Perceptual-cognitive expertise in sport: some considerations when applying the expert performance approach. Hum Mov Sci. 2005;24:283-307.

10. Brown S, Martinez MJ, Parsons LM. The neural basis of human dance. Cereb Cort. 2005;16:1157-67.

11. Malloy-Diniz LF, Fuentes D, Sedó M, Leite WB. Funções executivas. In: Fuentes D, Malloy-Diniz L, Camargo C, Cosenza R, editors. Neuropsicologia: teoria e prática. Porto Alegre: Artmed; 2008, p. 187-206.

12. Hogan M. Physical and cognitive activity and exercise for older adults: a review. Int J Aging Hum Dev. 2005;60(2):95-126.

13. Koechlin E, Corrado G, Grafman J. Dissociating the role of the medial and lateral anterior prefrontal cortex in human planning. PNAS. 2000;97(13):7651-6.
14. Ronström O. "I'm old an' I'm proud". Dance, music and the formation of cultural identity among pensioners in Sweden. The World of Music. 1994;3:5-30.

15. Lezak MD, Howieson DB, Loring DW. Neuropsychological assessment 4th ed. New York: Oxford University Press; 2004.

16. Shallice T. Specific impairments in planning. Philos Trans R Soc Lond Ser B. 1982;298:199-209.

17. Kricorian R, Bartok J, Gay N. Tower of London procedure: a standard method and developmental data. J Clin Exp Neuropsychol. 1989;16:840-50.

18. Shäfer S, Huxhold O, Lindenberger U. Healthy mind in healthy body? A review of sensorimotor-cognitive interdependencies in old age. Eur Rev Aging Phys Act. 2006;3:45-54.

19. Verghese J. Cognitive and mobility profile of older social dancers. JAGS 2006;54:1241-4.

20. Larson EB, Wang L, Bowen JD, McCormick WC, Teri L, Crane P, et al. Exercise is associated with reduced risk for incident dementia among persons 65 years of age and older. Ann Int Med. 2006;144:73-81.

21. Lautenschlager NT, Almeida OP. Physical activity and cognition in old age. Curr Opi Psychiatry. 2006;19:190-3.

22. Small GW, Silverman DH, Siddarth P, Ercoli LM, Miller KR, et al. Effects of a 14-day healthy longevity lifestyle program on cognition and brain function. Am J Geri Psych. 2006;14:538-45.

23. Kubesch S, Bretschneider V, Freudenmann R, Weidenhammer N, Lehmann $M$, Spitzer M, et al. Aerobic endurance exercise improves executive functions in depressed patients. J Clin Psychiatry. 2003;64:1005-12. 